\title{
ON THE DETERMINANTS OF A SUCCESSFUL, SUSTAINABLE-DRIVEN ADAPTIVE REUSE: A MULTIPLE REGRESSION APPROACH
}

\author{
DESPO S. PARPAS \& ANDREAS L. SAVVIDES \\ Department of Architecture, University of Cyprus, Cyprus.
}

\begin{abstract}
The purpose of this paper is to outline an ongoing research, examining the determinants of a successful, sustainable-driven development. The practice of adaptive reuse is connected with sustainable development and although it is widely believed that mainly economic factors drive possible development schemes, it is found through this research that, in the case of adaptive reuse, there are some other contributing criteria. The methodological tool implemented to obtain the results is multiple regression analysis and the contributions included in the model are based on the fields of socio-economics, culture and the environment. These vital contributions are key components of both the practice of adaptive reuse and sustainable-driven developments of the built environment. The advantage gained by applying statistical methods to examine multi-criteria cases is the possibility for well-justified observations; these are intended to be valuable tools for decision makers and involved stakeholders aiming to achieve successful sustainable adaptations. Although the findings presented in this paper are derived from research data collected in Cyprus, the methodological approach could be applied to a broader context, hence leading to more universal conclusions.

Keywords: adaptive reuse, multi-criteria analysis, multiple linear regression analysis, sustainabilitydriven development.
\end{abstract}

\section{INTRODUCTION}

Sustainable development has become a dominant force within the professions of architecture and urbanism; key points that derive from the prongs of economic, social, environmental and cultural frameworks are embraced. Moreover, this sort of analysis as it pertains to management and planning of underdeveloped or neglected sites within the existing built fabric incorporates tangible as well as intangible considerations. However, the emergence and quantification of different considerations are challenging when addressing the dynamic process of sustainable development; lifestyle trends, the state, the economy and the environment are constantly changing, thus altering the framework into which regeneration policies are developed and implemented.

Adaptive reuse as a practice has held a prominent role in several economic and cultural developments all over the world. Deliberate architectural and culturally appropriate adaptive reuse projects appear to be an entrepreneurial tool in achieving a sustainable-driven urban regeneration. Through corresponding initiatives, many architecturally significant buildings in historic cores have been preserved, abandoned areas have been upgraded into vibrant communities, multiple inactive units within the existing built fabric have gained new purpose and, simultaneously, both residents and visitors have been positively affected.

The ongoing research outlined in this paper brings together adaptive reuse and sustainable development by assessing its success through the use of 11 criteria emanating from the very prongs of sustainability. 


\section{ADAPTIVE REUSE AS A TOOL FOR SUSTAINABLE DEVELOPMENT}

\subsection{Defining Adaptive Reuse}

A simple definition for Adaptive Reuse is 'to re-use a building or structure for the purpose of giving it new life through a new function' [1] and it 'is described as developing the potential of additional use and wear for functionally obsolete buildings - it is essentially the recycling of a building' [2]. Ijla and Broström [2] appropriately separate adaptive reuse from restoration and renovation as these practices aim to restore buildings to a certain period or to upgrade them, respectively. Moreover, it is argued that adaptive reuse seeks to find a new use for the building. By the same token, Ewald [3] uses the term adaptive reuse without necessarily implying a change of use but generally as works including 'rehabilitation, renovation or restoration'. Adaptive reuse, semiotically, means to reuse in order to fit (from Latin Ad+aptar which means to+fit). However, what to fit is an interesting question because changes could occur in the needs of contemporary lifestyle, the new use, the climate, the physical or legislative context.

In contemporary times, older buildings are reused to fit contemporary lifestyle and changing needs. Most of the time, a new use is introduced to the existing shell, sometimes requiring restoration, upgrading or repairing works the degree of which depends on the case. Some adapted units are indeed recycled accommodating new uses and existing in the inherited built fabric; contemporary approaches were followed and viable solutions were found. Adaptive reuse concerns both highly important buildings historically (or museum pieces), and ordinary buildings and various housing typologies existing in the built context (Jane Jacobs also praised this approach as early as 1961), which is an essential aspect of the practice. However, not all buildings are good candidates for adaptation as their configuration and physical condition do not allow viable solutions. The main objective of a potential adaptation is for the buildings not to perform poorly but to meet the occupants' needs and to stand the test of time. Consequently, their useful life could be extended in a viable way, and possible adaptations could be seen as the medium to extend the useful life of buildings. Hence, their sustainable nature would also be embraced by corresponsive adaptations [2], possibly in an entrepreneur kind of way.

\subsection{Adaptive reuse as a proponent of sustainability-driven developments}

It can be shown that adaptive reuse can benefit both the local communities and the existing built fabric. It has positive attributes to: (1) socio-economic, (2) ecological-environmental and (3) cultural matters, as these are considered to be constituting the pillars of sustainability, adaptive reuse can potentially fall under its scope, as well.

Given that historic preservation is a great proponent of adaptive reuse, the following excerpt highlights the central argument of why adaptive reuse is considered to be a sustainable practice: 'Historic Preservation, in addition to being the ultimate form of recycling, plays a crucial role in preserving regional flavour while minimizing impacts on the environment' [3]. Undoubtedly, recycling participates in having an attitude towards a more sustainable way of living by taking more viable paths when it comes to materials and sources, and the grey energy associated with these.

In addition, potential abandonment is minimized along with its adverse effects on both the social and built fabrics. Several projects aiming to regenerate distressed areas through unit 
adaptation were presented as capitalizing on traditional cultural assets. Undoubtedly, financing is another critical player in adaptive reuse, 'but the financial and economic design is more than cost-it should be the subject of creative thinking. This can take the form of research into different funding models and partnerships and into how a project becomes viable' [1]. Economic opportunities could be catalytic to the decision-making process although, at the same time, all aspects of sustainable development should be considered.

Financial investment and human capital could be both secured with retaining the distinctive nature of certain places [4]. 'Core social values such as pride, memory and participation can all be enhanced by careful consideration of adaptive reuse strategies' [1], which highlights that adaptive reuse, in connection with the international charters praising the value of authenticity, contributes to maintaining the character and the vitality of the built fabric. Also, adaptive reuse, as a practice, involves contemporary means and approaches and this enhances the inherited value and helps to build up the heritage being left for future generations.

By connecting the new version of the building to its original character and the embedded narrative, the sense of place can be retained, and certain values are conserved (such as social, cultural and historical). Relevant links to the past and significant memories are kept and historical or cultural landmarks are safekept.

To sum up, a sustainable-driven development is geared to strengthen the ranks of residents and to spur economic growth. The aforementioned points have a direct impact on environmental, social and cultural matters but at the same time, they can have an indirect impact on economic ones, as well. More specifically, adaptive reuse can produce new visitor attractions (local or tourist) which have an effect on economic growth [5]-[7]. Hence, economic benefits, similar to all contributions, could exist at a range of scales (owner, community and urban scale).

\subsection{Life cycle and the notion of futurity in adaptive reuse}

The notion of futurity holds a key role for the research outlined in this paper as its meaning and the inclusion of the future generations address both fields of adaptive reuse and sustainability. Moreover, the notion of futurity is addressed directly in this research as it participates in assessing an adapted unit's success. Our 'common future' holds a prominent role in both fields of historic preservation and sustainable design. First, regarding historic preservation and as seen through the Declaration of Amsterdam (1975) and the Declaration of ICOMOS (1999), the 'common future' is highlighted. Arguably, the same expression appears in sustainability circles; 'Our Common Future' is the name of the publication of the United Nations, also known as the Brundtland Report, which was formulated in order to set 'a global agenda for change' [8]. The report also provides a definition which is as follows: 'Sustainability is to meet the needs of the present without compromising the ability of future generations to meet their own needs'.

Considering these established statements, adaptive reuse is indeed a sustainable practice; the continuous life cycle of a building is ensured as the building of interest is prevented from destruction [9]. The life cycle is connected with economic and environmental matters as well. First, the environmental load is lowered through the potential reuse of existing buildings, and this is connected with the environmental footprint and the grey energy of the buildings. In the assessment of the potential adapted asset, such matters should not be underrated. The building's grey energy is a crucial element as it revolves around energy consumption related to the transportations of materials and resources, construction or demolition works and the 
embodied energy of the materials. In addition, towards the environment, other benefits from adaptively reusing buildings include: decrease of carbon emissions and pollution as opposed to new constructions, minimization of demolition waste and reuse of contained energy, all of which have a positive effect on the affected communities [1], [9], [10]. Hence, the practice of adaptive reuse should be cherished and applied more thoroughly as this corresponds to the notion of recycling in the talk around sustainability.

\section{MULTI-CRITERIA ANALYSIS IN SEVERAL STUDIES}

\subsection{Use of multi-attribute frameworks}

There are several different approaches and categories used in assessing adaptive reuse. Concerning the latter, Urry [4] uses four categories: stewardship of the designated stock, investigation of the related space, visual consumption and economic exploitation. Other projects, where similar categorization is found, are Langston's ARP model [11] or Ding's AdaptStar [12], as well as, rating systems assessing a unit's performance (e.g. LEED, BREEAM).

Regarding the assessment of sites of interest, or potential cases of adaptive reuse, the most popular methodology used is the contingent valuation survey (CVS) which is based on economic valuation techniques of non-market resources and it is mostly used in environmental preservation. When it comes to preserving cultural heritage, the importance of such a technique lies in the measurement of the intangible aspects. Through the application of the willingness to pay model (WTP), some variables are seen in a tangible way for more efficient fiscal comparisons.

In all of the cases outlined in this section, several criteria derived from multi-attribute frameworks are used in the corresponding analyses, which are found to be crucial for all involved parties, as the necessity for well-substantiated results, and viable solutions, are recognised. The participating criteria in such theoretical or empirical projects, fall under the umbrella of sustainable development as the adaptive reuse of potential assets follows the route of sustainability.

The study of several assessment methods and popular rating systems was fundamental for the purposes of the ongoing research described in this paper. Valuable lessons were learnt in terms of finding the most appropriate methodological tool in the quest of the determinants of a successful adaptive reuse, and choosing the participating criteria themselves. The aim was to explore an evaluation process with universal application and several criteria covering different aspects of sustainability. In contrast to this, it was found that certain rating systems focus on green practices or incorrectly relate to sustainability. Nevertheless, such rating systems accelerate the assessed unit's upgrade, and promote a more viable living, which is useful, unless the implementation of these systems, as Parr [13] notes, aims at eco-branding.

\section{MULTIPLE REGRESSION APPROACH TO ADAPTIVE REUSE}

\subsection{Modelling approach}

This methodology was selected because regression analysis leads to weighted and welljustified results that illustrate the correlation of the selected variables with the adaptive reuse 
of a building. Moreover, the regression analysis seeks the assessment of a large number of built examples, which leads to better and more precise results within the framework of this research. In other methodologies, like the CVS and WTP models, only one unit is evaluated each time. More specifically, the CVS or WTP models deal with popular destinations or museum pieces. On the contrary, the aim of the project outlined in this paper was to deal with all kinds of buildings, museum pieces or plain and ordinary buildings.

The multiple linear regression analysis is based on a simple regression analysis, and it has the following general form:

$$
y_{i}=\beta_{0}+\beta_{1} x_{1 i}+\ldots+\beta_{k} x_{k i}+u_{i}
$$

where ' $y$ ' is the dependent variable and it is explained by all ' $x$ 's whereas ' $i$ ' indicates each observation. The regression does not have only one regressor but has ' $k$ ' regressors/independent variables. In the case of the empirical study outlined in this paper, the regression has 11 regressors. The intercept $\beta_{l}$ is fundamental for the model because it is the medium to show how powerful the relationship between ' $y$ ' and the different regressors is, and the degree to which the variable ' $y$ ' will change with a possible change in the value in ' $x$ '.

Multiple regression analysis has been implemented in other empirical projects relevant to adaptive reuse, as well. First, the sustainable status of realized projects was tested against several criteria, economic growth, total resource consumption, labour hours and resource productivity [14]-[16].

Moreover, multiple regression analysis was applied in order to determine the statistical relationship between social sustainability and adaptive reuse [17]. The criteria for this study were associated with matters of culture, aesthetics, society and human comfort. The run of the model revealed that sustainable behaviour and perseverance of the heritage significance were correlated. However, the sample used in order to draw these conclusions was rather small.

Similarly, however, Briggs [18], created a large directory, and used the same methodology to examine textile mills, and more specifically, the locational aspect of their adaptive reuse. The probability of the reuse was established, which was crucial as the research was conducted in order for an existing situation of abandonment, and underuse of existing shells, to change. This approach was successful because it could be reapplied elsewhere, for a different building typology, and new directories. In addition, the findings of such a study could prove helpful to stakeholders, or other involved parties, as the inactive textile mills, or other inactive units, could be regenerated through new redevelopment proposals and investments.

Shipley's [19] research, on the other hand, was seeking to examine whether designation of historic buildings is negatively correlated with property values using regression analysis. This research revealed that historic designation has no negative effect on property values. On the contrary, the research showed that 'the rate of sale among designated properties is good or better than ambient market trends'. By the same token, Leichenko et al.'s [20] paper suggests that historic preservation is positively correlated with high property values. The results were formulated after regression analysis was conducted with data contributions from cities in Texas.

Moreover, Young [21] through his paper proposes the use of regression analysis as being helpful in calculating the correlation between adaptive reuse and displacement rates in Los Angeles. This is because it is evident that land use is significantly driven by development incentives and city regulations. In his proposal, it is noted that the context and events in 
Los Angeles are 'non-exclusive', and therefore, the potential for the application of such a method is crucial to investigating gentrification and smart growth throughout the United States. The results could encourage adaptive reuse and could inform existing regulations and policies.

Nevertheless, a regression analysis has never been developed and conducted in order to establish the criteria that mostly affect a successful adaptive reuse, which is essentially the main objective of the research presented in this paper.

\subsection{The dependent variable of the model}

The dependent variable (or ' $y$ ') of the model used to meet the goals of the research presented herein reflects the success of the adaptation. In establishing how successful a reused building is, it is important to establish the possible fields pertaining to this. A successful adaptation could be based entirely on the quality of the restoration works; on general perception; on the popularity of a building's new use; and/or on the relationship in the active years of each use. Some of the aforementioned topics could be biased, and therefore for this study, the active years of a use were chosen as a given variable existing in the real world that might be correlated effectively with several independent variables that would result in explaining the successful components behind such adaptations. Consequently, the dependent variable ' $y$ ' is, fundamentally, an index that establishes the degree of how successful a rehabilitation is and its formula has the following form:

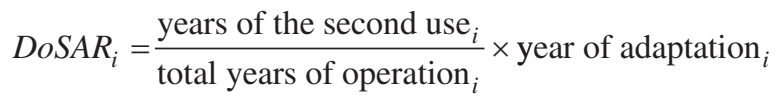

where 'DoSAR' is the 'degree of a successful adaptive reuse' and it is the dependent variable of the model, and ' $i$ ' indicates each case study.

\subsection{The independent variables of the model}

The independent variables chosen for the model, having possible explanatory power on the dependent variable, derive from critical literature review of other empirical projects and assessment methodologies, as well as from site visits to adaptively reused units.

The independent variables fall into three categories: physical, economic and utilitarian. More specifically, the 11 independent variables are the following:

\subsubsection{Construction era}

This variable could help draw conclusions on whether legislative, historical or cultural aspects are correlated with a successful adaptive reuse.

Specifically, what was important to examine here was whether signs of early informal practices have an important role in making a contemporary adaptation successful. Up until Ottomans rule and before the British colonization of Cyprus, these practices were dominating the scene. However, after the colonization of the island and the launch of large development schemes or the extensive spread of bureaucracy, and after the introduction and wide use of concrete in Cyprus, the informal practices started to deteriorate and, eventually, to disappear. Consequently, the construction era was included in the model in order to examine whether the informal practices affect contemporary 
adaptations. The year 1925 was set as the base year, since Cyprus became a Crown Colony in 1925.

A hypothesis here (or a possible outcome) is that the variable 'construction era' has a positive correlation with the dependent variable ' $y$ ' or the DoSAR index, as it would be expected that if a property was developed during formal practices, then owners or decision makers would proceed to a potential adaptive reuse because of more extensive original works. On the other hand, there could be a negative correlation as well, whereby stakeholders might show more sensitivity towards older structures because of their authentic indigenous aesthetics.

\subsubsection{Number of usual users}

The number of usual users reflects the type of activities or uses that are introduced into an old shell. For instance, a small number in the input means residential or small-scale activities such as offices whereas a bigger number indicates a more public or commercial use.

When a building is publically accessible, then the number of frequent users could imply the popularity of the new use, and in this case, the two variables would have a positive correlation. On the other hand, if a building was never designed to host a large number of people, but the new use proposes such a condition, then the building could be driven to obsolescence more rapidly. In this case, the two variables might be negatively correlated.

\subsubsection{Location}

The location's input may indicate whether a building in a historic city centre is more likely to be adaptively reused. Moreover, 'Location. Location. Location' is a popular motto in Real Estate circles. Therefore, this parameter was selected in order to investigate whether location holds a leading role in the process of adapting an old building. At this point, the location is distinguished by the buildings' siting in a rural or urban context. This is expressed by dummy variables; 1 for urban and 0 for rural. A hypothesis here is that 'Location' is positively correlated with a success rate in adaptive reuse.

\subsubsection{Gross domestic product growth rate}

The gross domestic product growth (GDPG) rate is the value pertaining to wealth of the state at a specific time. The more the wealth, the more the intention to develop, or redevelop in this case, a property. For this reason, this input was selected to establish whether a wealthy society tends to reuse its building stock. Moreover, if the society shows signs of prosperity, this could mean that the adaptation works are of better quality and therefore, the second use has the advantage over the former use because of this aspect. As explained above, it is expected that a higher value in the GDPG could be associated with higher values in the index for a successful reuse, which means that the two variables could be positively associated.

\subsubsection{Real cost per square metre}

The cost of the adaptation could act as a barrier in some cases whereas in others it could be encouraging. Due to inflation, the cost should be converted to reflect the real cost for better comparisons. This is because the purchasing power changes, and everything should be calculated (and examined) on a common basis, since this research examines different conversions take place at different periods in time. More capital might mean construction works of better quality, or more flexibility to produce a more appealing product. The two variables could be positively correlated. 


\subsubsection{Price index of the construction materials}

The price index of the construction materials, accounting for inflation, could have an impact on the variety of rehabilitations and the quality of the work that might occur. High values of the price index of the construction materials at the time of the work could mean that materials are tougher to purchase, and that perhaps the adaptation work suffer from market forces. Therefore, a possible scenario is that this independent variable is negatively correlated with the dependent variable 'DoSAR'.

\subsubsection{Primary construction material}

The primary construction material is an important variable as it reflects a building's life expectancy and/or embodied energy (the data for these were obtained from scientific databases). The materials' properties mentioned here could affect several aspects of the rehabilitation process, such as the users' comfort, and the life span of the building leading to the possibility of long-term successful reuse. The use of the variable itself could indicate the importance it holds statistically when evaluating the degree of a successful reuse. This comparison could prompt deliberation around the debate on whether, for example, to adaptively reuse concrete buildings, and if the use of non-organic materials is positively correlated with successful adaptive reuse.

\subsubsection{Type of use}

The different uses could affect the successful rehabilitation of a building as a unit as well as the neighbourhood of which it is part. The different types of use that could have an effect on the index are: (1) multi-use, (2) industrial, (3) educational, (4) cultural, (5) institutional, (6) commercial/services, (7) hospitality, (8) residential, (9) office, (10) health and (11) religious. However, for the purposes of the regression, a dummy variable could be used, and therefore, the input was boiled down to ' 0 ' for residential and ' 1 ' for non-residential. The use of the variable may reveal if the introduced use is statistically significant. Then, the difference in use may be examined to establish which type of use is positively associated with the dependent variable 'DoSAR'.

\subsubsection{Viability score}

After reviewing relevant theory on rating systems measuring the sustainable or green behaviour of reused or retrofitted buildings, a rating system was constructed for the purposes of this research. The rating system consists of several binary questions, which is a more direct and easily applicable way to rate a building's viability and sustainable behaviour. Moreover, this does not require third-party evaluation, and it does not rely on subjective judgements and decisions, but on observations. The viability index includes four categories that examine a building's state regarding relevant environmental actions, management, historical and cultural aspects and social matters.

This variable's value is the achieved score of the fabricated check list, and is indicated by a real number, which is the degree of a viable adaptation. Therefore, the regression could reveal the relationship between the degree of a successful reuse and the degree of a sustainable attitude. The question to be articulated here is whether a successful rehabilitation could depend on its sustainable character, as given by the rating system utilized. A possible outcome is that the index reflecting attitude towards sustainable living is positively associated with the degree of successful reuse, meaning that high values in the first variable would be associated with high values in the latter variable. 


\subsubsection{Extension and change in use}

After all the site visits and the relevant research concerning all case studies, it was decided that these two final variables could be included in the model. A variable concerning a contemporary extension could be positively correlated with the degree of success of the reuse as it deals with the unit's performance, and its conformance to a user's growing needs. On the other hand, a variable concerning a change in a proposed adaptive use could be both positively and negatively correlated with the adaptation's success. Depending on the surrounding context, and the urban fabric's requirements, or the user's lifestyle, a change in use could be beneficial, or it could be proven unsuccessful, with the unit ceasing its operation. Nevertheless, it would be interesting to explore the most successful cases of adaptively reusing obsolete buildings and to examine if the change in use plays an important role.

\section{ANALYSIS AND FINDINGS}

The research project accounts for 100 case studies from Cyprus (Fig. 1). Fundamentally, this acts as a pilot that might prompt further research beyond the borders of the island. The multiattribute framework of the project, similar to other projects, celebrates the uniqueness of each case study and the realization that each is characterized by a particular identity carrying a particular story in their own right. Moreover, the same model has the potential to be applied elsewhere.

The most striking information after running the regression model is that there are only two independent variables that cannot be omitted from the model, with high explanatory power over the degree of a successful adaptive reuse. These are: the construction era of the asset and the price index of the construction materials at the time of the conversion. In contrast, all other independent variables can be omitted from the model. Furthermore, the original primary construction material, change in use, and the number of usual users have negligible explanatory power over the DoSAR.

In the case of the price index of the construction materials, the predictions were correct; high values in the price index means that materials are tougher to purchase, and this may have

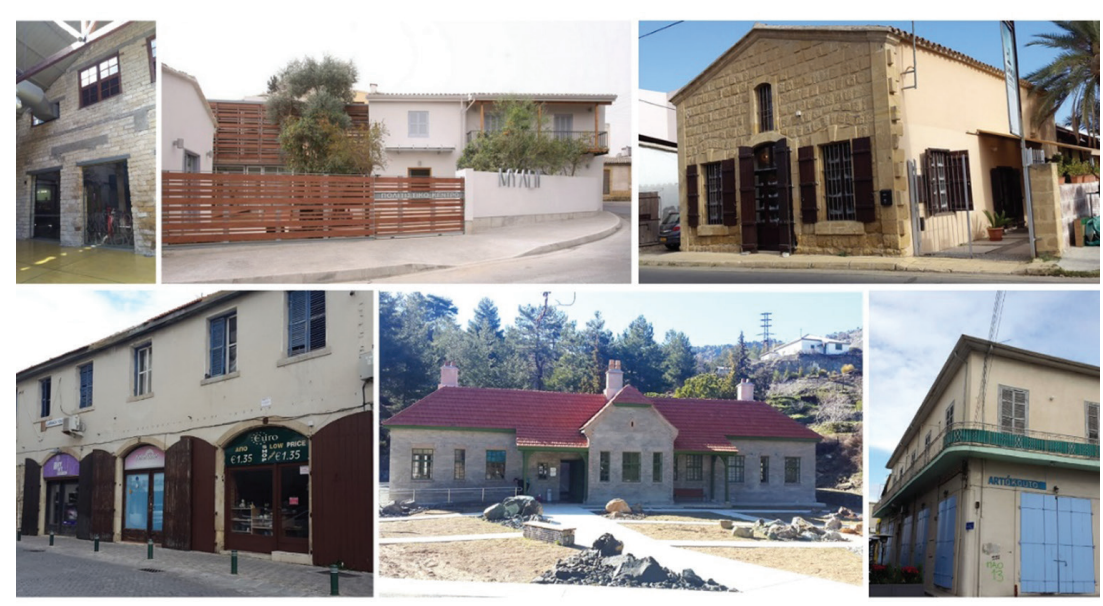

Figure 1: Buildings of different typologies, uses, sizes, construction materials and construction eras constituting the sample. 
a negative effect on the construction work. Therefore, it is evident through this project that the two variables are negatively correlated. As far as the construction era is concerned, it is revealed that in Cyprus, both historical and legislative backgrounds hold a key role in the practice of adaptive reuse. More specifically, it is proven that buildings constructed after the establishment of the Crown Colony hold an advantage when a successful reuse is at stake.

It is also interesting to explore some important criteria that are found in the middle of the significance scale. These are the location, the GDPG rate, the real cost of the adaptation and the achieved viability score. Concerning location, although it is proven as being less significant in this research, it is found to be statistically significant in other studies [18]. In addition, the adapted units located in an urban context hold a significant advantage over ones located in rural areas which means that, in Cyprus, the useful life of the assets is more likely to be extended in urban areas.

Moreover, the GDPG rate, although it is a great indicator for a region's economic growth, is proven not important statistically in the case of Cyprus. Similarly, the real cost was expected to have high explanatory power over the DoSAR, and a positive correlation was part of the hypothesis, yet this was overturned. What this means essentially is that, in Cyprus, the literal costs are uncorrelated with a successful reuse, or the extension of a unit's useful life.

As far as the viability index is concerned, the negative value of the coefficient reveals the negative relationship between this parameter and the degree of a successful reuse. Contrarily enough, it was expected that the viability index showcasing a positive attitude towards sustainable living would be positively associated with a successful reuse (meaning that high values in the first variable would be associated with high values in the latter variable) (Table 1).

Finally, another parameter can exist in such investigations, though it cannot be measured easily nor can it be predicted. It is the human factor. People are unpredictable, changing

Table 1: Expected vs. real correlations between the dependent variable and the independent variables.

\begin{tabular}{lcc}
\hline Independent variable & $\begin{array}{c}\text { Expected correlation with } \\
\text { DoSAR }\end{array}$ & $\begin{array}{c}\text { True correlation with } \\
\text { DoSAR }\end{array}$ \\
\hline Construction era & $+/-$ & + \\
Number of usual users & $+/-$ & + \\
Location & + & + \\
GDPG rate & + & + \\
Real cost per m ${ }^{2}$ & + & - \\
Price index of the construc- & - & - \\
tion materials & & + \\
Primary construction mate- & $+/-$ & + \\
rial & & - \\
Type of use & $+/-$ & - \\
Viability score & + & + \\
Extension & + & \\
Change in use & $+/-$ & \\
\hline
\end{tabular}


and progressing, and therefore, when it comes to introducing a new use in an existing shell, the choice itself could be equally proven a success, or a failure; extending the unit's useful life, or ending it. Trends, as well, change all the time; fashionable uses and places emerge and disappear. The trends in the general market could influence involved parties to gainfully exploit existing built capital rather than building something new (see also [10], [22]). Perhaps the human factor and each person's uniqueness, and free will, in combination with the changing contemporary trends, are difficult to be quantified and inserted in a model.

\section{CONCLUSIONS}

The notion of futurity is present in both adaptive reuse and sustainability; by reusing inactive shells in viable ways, the useful life of existing building stock is extended, and sustainable concepts are strengthened. Bullen [10] suggests that old buildings should be treated as a reusable source and not as a product because most products are consumed and then they become waste. The purpose of this paper was to investigate the strong connection between adaptive reuse and sustainable development and to explore the most important variables to a successful adaptive reuse following a sustainable route. The project presented herein depends on data obtained from Cyprus, with criteria derived from economics, ecology, society and preservation of ethics.

The run of the model provided evidence that economics alone cannot drive a successful sustainable-driven adaptive reuse, although some scholars (e.g. [23]-[25]) argue that sometimes economic matters are prevalent when sustainable development is at stake. Adaptive reuse is part of a sustainable approach to development, and it incorporates salient aspects of sustainability, yet there are projects where money is not the main driving force when it comes to a successful reuse, or sustainable re-development [17], [26]. However, one of the two most significant variables is the price index of the construction materials, which does emerge from economics. On the other hand, the relevant literal costs have negligible explanatory power over the DoSAR, which may be promising in the field of adaptive reuse. In the case economic concerns are prevalent, this could be both limiting and worrisome, especially in the decisionmaking process of whether to reuse a unit or not, and consequently fiscal concerns should not be perceived as a strict barrier. On the contrary, this study reveals that there can be other critical variables as well, although not as prominent. More specifically, the construction era is highly connected with the legislative background, and the settlement development in the case of Cyprus. Consequently, the decisions made after weighting formal and bureaucratic processes, as opposed to informal and silent actions, may significantly affect the success of a potentially adapted building.

In summary, there may be economic, physical, legislative and utilitarian criteria that affect an adaptation positively. However, their contributions to achieving viable solutions can be unequal. It is crucial to work through multi-attribute frameworks, and to monitor the general picture consistently in order to safekeep the built heritage. Such actions have a positive effect on both individuals and the general public, as diachronic and cohesive environment subdues feelings of insecurity and displeasure, and energy-related consumption, time and costs are minimized. The aforementioned points are some of the reasons adaptive reuse is considered sustainable as well.

The findings of this research and other empirical studies are based upon data collection and analysis of the practice of adaptive reuse. The correlations among the several parameters or criteria in various regions could possibly affect decision making when it comes to the decision of whether to adapt a building or not. Cost-benefit methodologies and multiple regression 
models provide insights into the trends of present best practices in adaptive reuse and therefore, a diverse spectrum stakeholders could benefit from such studies; policymakers could have a more broad-based knowledge on interdependent issues concerning adaptive reuse and their approaches could be more ethical and their actions more holistic when proposing a sustainable future, with certain areas of great potential for redevelopment earmarked to be retained and refilled with life.

\section{REFERENCES}

[1] ODASA, Adaptive Reuse: ODASA Design Guidance Note, 2014.

[2] Ijla, A. \& Broström, T., The sustainable viability of adaptive reuse of historic buildings: the experiences of two world heritage old cities; Bethlehem in Palestine and Visby in Sweden. International Invention Journal of Arts and Social Sciences, 2(4), pp. 52-66, 2015.

[3] Ewald, C.R., The New Western Home, Gibbs Smith: Salt Lake City, 2007.

[4] Urry, J., Consuming Places, Routledge: London, 1995.

[5] Newby, P.T., Tourism: support or threat to heritage? Building a New Heritage: Tourism, Culture and Identity in the New Europe, eds. G.J. Ashworth \& P.J. Larkham, Routledge: London, 1994.

[6] Strange, I., Local politics, new agendas and strategies for change in English historic cities. Cities, 13(6), pp. 431-437, 1996. https://doi.org/10.1016/0264-2751(96)00030-3

[7] Ashworth, G.J. \& Voogd, H., Marketing the city: concepts, processes and Dutch applications. Town Planning Review, 59, pp. 65-80, 1988. https://doi.org/10.3828/ tpr.59.1.9t614v1537652w31

[8] World Commission on Environment and Development (WCED). Our Common Future, p. 2, 1987.

[9] Mohamed, N. \& Alauddin, K., The criteria for decision making in adaptive reuse towards sustainable development. Proceedings of the MATEC Web Conferences 66, 2016.

[10] Bullen, P., Adaptive reuse and sustainability of commercial buildings. Facilities, 25(1/2), pp. 20-31, 2007. https://doi.org/10.1108/02632770710716911

[11] Langston, G., Validation of the adaptive reuse potential (ARP) model using icon CUR. Facilities, 30(3/4), pp. 105-123, 2012. https://doi.org/10.1108/02632771211202824

[12] Ding, G., Sustainable construction: the role of environmental assessment tools. Journal of Environmental Management, 86(3), pp. 451-464, 2008. https://doi. org/10.1016/j.jenvman.2006.12.025

[13] Parr, A., Hijacking Sustainability, MIT Press: Cambridge, 2012.

[14] Bockermann, A., Meyer, B., Omannc, I. \& Spangenberg, J., Modeling sustainability: comparing an econometric (PANTA RHEI) and a systems dynamics model (SuE). Journal of Policy Modeling, 27(2), pp. 189-210, 2005. https://doi.org/10.1016/j.jpolmod.2004.11.002

[15] Cirman, A., Mandic, S. \& Zoric, J., What determines building renovation decisions. Proceedings of the ENHR Conference, Toulouse, 2011.

[16] Fujita, Y., Assessment of $\mathrm{CO} 2$ emissions and resource sustainability for housing construction in Malaysia. International Journal of Low-Carbon Technologies, 4, pp. 16-26, 2009. https://doi.org/10.1093/ijlct/ctp002

[17] Basha, B., Viability of adaptive reuse with social approach. Presented in Fulfillment of the Requirement for the Degree of Master in Science. International Islamic University of Malaysia, 2016. 
[18] Briggs, J.M., Locational aspect of adaptive reuse the case of North Carolina's textile mills. Presented to the Faculty of the Department of Geography East Carolina University in Partial Fulfilment of the Requirements for the Degree Master of Arts in Geography. East Carolina University, 2010.

[19] Shipley, R., Heritage designation and property values: is there an effect? International Journal of Heritage Studies, 6(1), pp. 83-100, 2000. https://doi. org/10.1080/135272500363760

[20] Leichenko, R.M., Coulson N.E. \& Listokin, D., Historic preservation and residential property values: an analysis of Texas cities. Urban Studies, 38(11), pp. 1973-1987, 2001. https://doi.org/10.1080/00420980120080880

[21] Young, M.A., Adapting to adaptive reuse: comments and concerns about the impacts of a growing phenomenon. Southern California Interdisciplinary Law Journal, 17, pp. 703-728, 2009.

[22] Bullen, P., Adaptive reuse and sustainability of commercial buildings. Facilities, 25(1/2), pp. 20-31, 2007. https://doi.org/10.1108/02632770710716911

[23] Worster, D., The shaky ground of sustainability. Global Ecology: A New Arena of Political Conflict, ed. W. Sachs, Furnwood Publishing: Canada, pp. 132-145, 1993.

[24] Dong, X., Zhang, J., Zhi, R., Zhong, S. \& Li, M., Measuring recreational value of world heritage sites based on contingent valuation method: a case study of Jiuzhaigou. Chinese Geographical Science, 21(1), pp. 119-128, 2011. https://doi.org/10.1007/ s11769-011-0445-5

[25] Throsby, D., Determining the value of cultural goods: how much (or how little) does contingent valuation tell us? Journal of Cultural Economics, 27(3/4), pp. 275-285, 2003. https://doi.org/10.1023/a:1026353905772

[26] Parpas, D. \& Savvides, A., Sustainability driven adaptive reuse: evaluation of criteria in a multi-attribute framework, WIT Transactions on Ecology and the Environment, 217, WIT Press 2018, ISSN 1743-3541. 